\title{
Pilotage d'un programme visant à sécuriser l'espace et à renforcer les ressources des adolescentes vivant dans les zones urbaines du Ghana
}

Sarah Engebretsen

Population Council

Selina F. Esantsi

Population Council

Follow this and additional works at: https://knowledgecommons.popcouncil.org/departments_sbsr-pgy

Part of the Demography, Population, and Ecology Commons, Family, Life Course, and Society Commons, Gender and Sexuality Commons, International Public Health Commons, and the Medicine and Health Commons How does access to this work benefit you? Let us know!

\section{Recommended Citation}

Engebretsen, Sarah and Selina F. Esantsi. 2011. "Pilotage d'un programme visant à sécuriser l'espace et à renforcer les ressources des adolescentes vivant dans les zones urbaines du Ghana," Promouvoir des transitions vers l'âge adulte saines, sécurisées et productives Resumen no. 40. New York: Population Council. 


\section{Pilotage d'un programme visant à sécuriser l'espace et à renforcer les ressources des adolescentes vivant dans les zones urbaines du Ghana}

\section{Préparé par Sarah Engebretsen et Selina Esantsi}

$$
\text { L: }
$$

a population du Ghana compte 24 millions d'habitants et devrait atteindre 31,8 millions d'habitants en 2025-soit une augmentation de 32 pour cent sur 15 ans (PRB 2010). Cette croissance démographique va de pair avec l'urbanisation rapide sachant que les populations se déplacent vers les grandes villes, notamment à Accra et Kumasi. Alors que la migration vers une zone urbaine présente souvent de nouvelles opportunités, la vie urbaine peut être très sombre pour les adolescentes. Les adolescentes qui vivent en milieu urbain connaissent rapidement une diminution du taux de scolarisation, souvent parce qu'elles doivent apporter un soutien économique à leur famille. Même si le taux de scolarisation est de 92 pour cent pour les adolescentes âgées de 11 ans vivant en milieu urbain, ce taux chute à 51 pour cent chez les adolescentes âgées de 17 ans (Population Council 2010). En outre, près de 4 pour cent des adolescentes âgées de 10 à 14 ans en milieu urbain ne sont pas scolarisées et ne vivent avec aucun de leur parent (Population Council 2010). Les filles qui vivent à l'écart de leur famille et qui ne sont pas scolarisées sont souvent dépourvues de réseaux sociaux et ont peu d'occasions d'acquérir des compétences nécessaires à la vie adulte. L'acquisition de ces compétences est importante pour le développement du Ghana compte tenu de sa population de plus en plus jeune, 39 pour cent des habitants sont âgés de moins de 15 ans (PRB 2010).

Le Population Council dispose d'un portefeuille mondial d'activités consacrées aux adolescentes vulnérables. Bien que chaque programme soit spécifique à un contexte, tous apportent une plateforme sûre et axée sur les filles dans laquelle les adolescentes peuvent développer des compétences de base, nouer des amitiés, recevoir et apporter un soutien par les pairs, accroître leurs réseaux sociaux et bénéficier

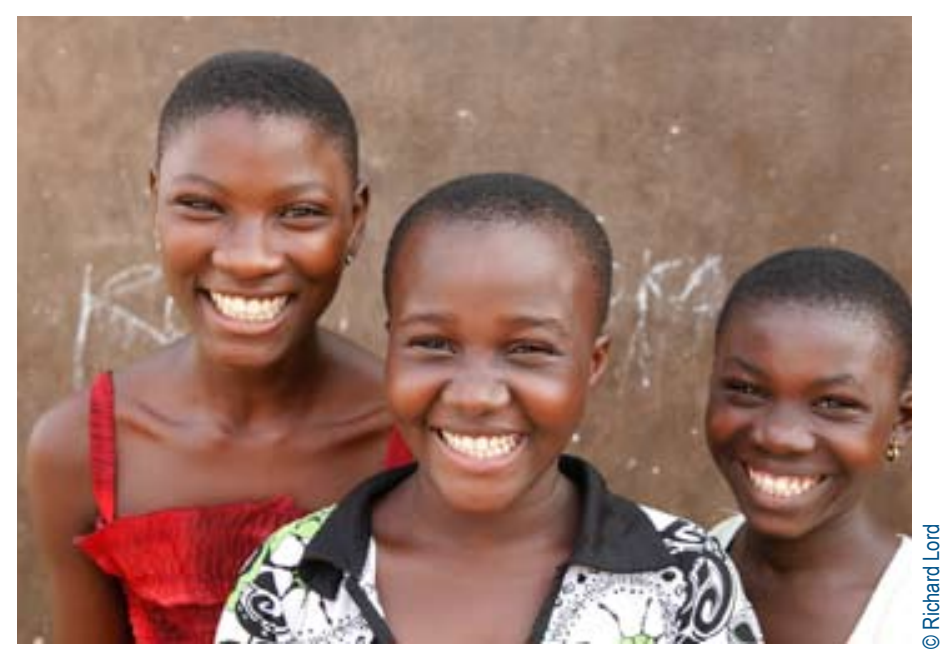

d'un encadrement par des adultes de confiance qui peuvent plaider en faveur des filles (Brady 2003). Le Population Council s'est appuyé sur l'expérience d'un projet de santé communautaire à Accra pour sélectionner le site approprié d'un projet pilote avec des adolescentes dans les zones urbaines du Ghana.

En 1999, le Service de santé du Ghana a adopté un modèle de prestation de services de santé communautaire fondé sur l'important travail réalisé par le Population Council à Navrongo et Nkwanta. Connue sous le nom d'Initiative à base communautaire de planification et services de santé (CHPS), cette intervention visait non seulement à fournir des soins de santé cliniques aux femmes et aux enfants, mais également à faciliter l'accès aux services de planification familiale. 
Initialement conçu comme un programme rural, le CHPS a ensuite été mis à l'essai dans deux communautés urbaines de la région du Grand Accra : le quartier U-Compound dans la municipalité de Téma et le quartier Glefe dans la métropole d'Accra. La municipalité de Téma est un grand port et le quartier U-Compound est une communauté de pêcheurs située sur l'eau. Les moyens de subsistance des habitants du quartier U-Compound dépendent de la pêche et de la vente des produits de la mer aux commerçants qui travaillent dans les marchés de Téma. Les données du CHPS relatives au quartier U-Compound révèlent que cette implantation urbaine surpeuplée a une population de 3585 habitants, qui vit dans des bâtiments de fortune et souffre de mauvaises conditions d'hygiène. L'expérience du CHPS en milieu urbain a révélé que les adolescentes du quartier U-Compound manquaient de connaissances en santé sexuelle et reproductive et n'avaient pas accès aux services disponibles. Bien que les parents se préoccupent des problèmes de grossesse, ils ne se sentent pas munis des outils nécessaires pour aborder les questions de santé sexuelle et reproductive avec leurs filles. Compte tenu des besoins des adolescentes à Téma et de l'expérience du Population Council dans cette implantation urbaine informelle, le quartier U-Compound a été sélectionné comme site pour la mise en place d'un programme pilote sur le renforcement des ressources sanitaires, sociales et économiques des adolescentes.

\section{Collaboration avec la communauté pendant la phase préliminaire de mise en œuvre}

Au cours des mois qui ont précédé le lancement du programme, l'équipe du projet s'est réunie avec les parties prenantes, y compris les fonctionnaires du Service de santé du Ghana, l'équipe de gestion sanitaire de la municipalité de Téma, le ministère des Femmes et des Enfants, et le ministère de l'Emploi et des Affaires sociales. Des consultations ont également eu lieu avec les agents de santé communautaire et les bénévoles des quartiers urbains du CHPS, les aînés des groupes ethniques représentés dans le quartier U-Compound et le chef de la municipalité de Téma. Les rencontres avec ces personnes ont joué un rôle essentiel dans le processus d'entrée de la communauté. Un durbar (réunion de sensibilisation communautaire) a été organisé pour présenter le programme et engager les membres de la communauté-en particulier les parents, les aînés, les chefs et les enseignants communautaires et les filles-dans les discussions visant à obtenir leurs observations sur les besoins des adolescentes vivant à U-Compound. Les discussions ont révélé la nécessité de mettre en place un programme dans lequel des femmes de confiance dispenseraient une instruction en matière de santé sexuelle et reproductive et dans d'autres domaines importants pour assurer une transition saine vers l'âge adulte. Des jeunes leaders femmes ont ensuite été sélectionnées pour être les tutrices du programme. Une attention particulière a été accordée à la sélection des tutrices qui provenaient du site d'intervention et qui étaient un peu plus

âgées que la population cible, afin que les filles participant au programme puissent s'identifier à elles, se sentir à l'aise pour les approcher et obtenir des conseils. Les tutrices ont été formées pour promouvoir la santé des filles et leurs opportunités économiques et sociales à l'aide d'un programme flexible axé sur la demande. Bien que le contenu du programme était structuré, les tutrices ont également appris à adapter les séances en fonction des intérêts et des aptitudes des filles.

Les discussions communautaires ont révélé que la présence des représentants communautaires était nécessaire pour servir de lien entre les tutrices du programme et l'ensemble de la communauté. L'équipe du programme a établi le rôle des mères de la communauté et a choisi quatre femmes qui inspirent le respect des filles et des autres membres de la communauté. Les mères communautaires sont responsables de la médiation des problèmes potentiels pouvant résulter de la partici-

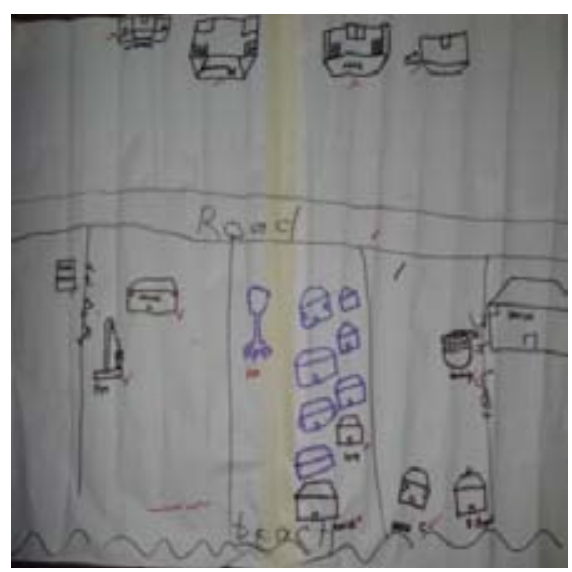

Utilisation de la cartographie participative, les filles ont identifié les espaces sûrs et dangereux dans leur communauté. pation d'une jeune fille dans le programme et du suivi avec les familles au cas où une fille cesserait d'assister au programme.

Le recrutement a été réalisé avec la contribution des aînés, des agents de santé communautaires et des bénévoles en santé communautaire. Ces intervenants ont permis d'identifier les filles dans le groupe cible : les filles âgées de

12 à 15 ans. Sachant que le quartier U-Compound est une implantation informelle de petite taille, l'équipe du projet a été en mesure de faire du porte-à-porte pour identifier les ménages dont les membres répondaient aux critères de sélection. Le consentement éclairé a été demandé aux filles intéressées et à leurs parents, et les entretiens d'admission ont été menés avec les participantes au programme. Les questions posées pendant ces entretiens ont porté sur les connaissances, les attitudes et les comportements liés au capital social, à l'éducation, aux compétences de la vie courante, à la santé, à la santé sexuelle et reproductive, et aux aspirations des filles pour leur avenir.

La prochaine étape était de trouver un espace dans la communauté où les groupes de filles pourraient se rencontrer. II est important d'avoir un espace où les filles se sentent en sécurité et ont un certain niveau d'intimité afin qu'elles puissent s'exprimer sans gêne et discuter de leur vie avec d'autres adolescentes. L'équipe du projet a commencé par faire un exercice de cartographie participative avec les filles afin d'évaluer leur perception sur la sécurité de différents endroits dans la communauté. Les filles ont déclaré être préoccupées par le fait que des 


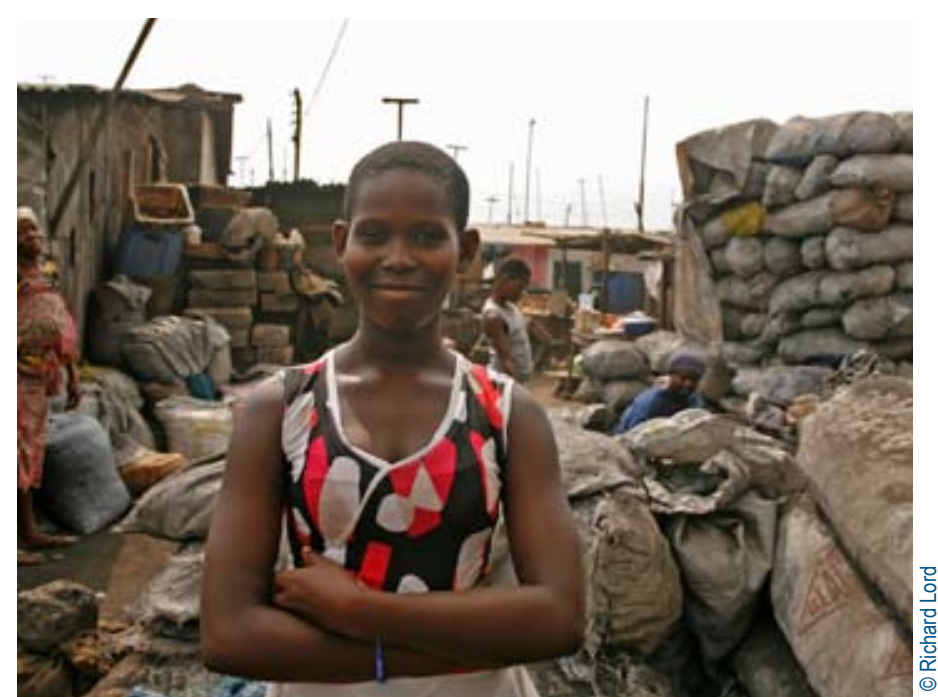

Le recrutement dans le programme comprenait des visites en porte à porte dans la communauté pour identifier les filles admissibles âgées de 12 à 15 ans.

\section{Perspectives communautaires sur le programme}

"Grâce aux leçons sur l'éducation financière, je comprends maintenant l'importance de l'épargne, et avec l'aide de l'une de nos tutrices, j'ai ouvert un compte à la banque. Je fais des économies dans le but d'étudier. Je veux être infirmière plus tard."

\section{- Participante au Programme}

"Je me suis fait de nouvelles amies et je comprends maintenant comment mon corps fonctionne. Toutes mes amies ont leurs premières règles depuis quelques mois et j'avais peur

d'avoir un problème. Mais maintenant je sais que cela se produit à des moments différents chez toutes les femmes et que le mien viendra bientôt."

\section{- Participante au programme}

"Depuis que ma fille a commencé le programme, j'ai remarqué qu'elle m'informe ou me demande la permission à chaque fois qu'elle doit sortir. Avant le programme, elle ne le faisait pas."

\section{- Mère d'une participante au programme}

"Je pense que le programme fait du bon travail avec les filles. Elles prennent de plus en plus conscience de leurs actions. Elles semblent apprécier les séances et sont toujours pressées d'y assister."

$$
\text { - Aîné de la communauté }
$$

lieux publics sélectionnés, tels que des abris communautaires, étaient fréquentés presque exclusivement par des hommes et n'étaient pas des lieux sûrs pour elles. Elles ont déclaré se sentir très en sécurité dans des églises et des écoles. Des discussions ont eu lieu avec le directeur de l'école communautaire, qui a mis à la disposition du programme une salle inoccupée. Les adolescentes de la communauté ont passé une journée à nettoyer et à décorer cette pièce dans le but de s'approprier un espace réservé uniquement à des filles.

\section{Un programme pilote visant à promouvoir les compétences} financières, sanitaires et de la vie courante chez des adolescentes vivant en milieu urbain À la fin de l'année 2009, le Population Council a lancé une intervention pilote destinée aux adolescentes vivant dans le quartier U-Compound. Ce programme pilote visait à tester la faisabilité du lancement d'une intervention axée sur la création d'espaces sécurisés pour renforcer les ressources dans les zones urbaines du Ghana. Plus précisément, le projet pilote est destiné à déterminer la possibilité de faire participer les filles et leur communauté dans ce type de programme, de trouver des femmes leaders expérimentées au sein de la communauté et de concevoir un programme multidisciplinaire pour répondre aux besoins des bénéficiaires du programme. Alors que les interventions traditionnelles destinées aux jeunes sont souvent fondées sur la connaissance des programmes de santé reproductive, l'équipe de ce projet a cherché à établir une intervention à large assise qui s'appuie sur les ressources sanitaires, sociales et économiques des adolescentes.

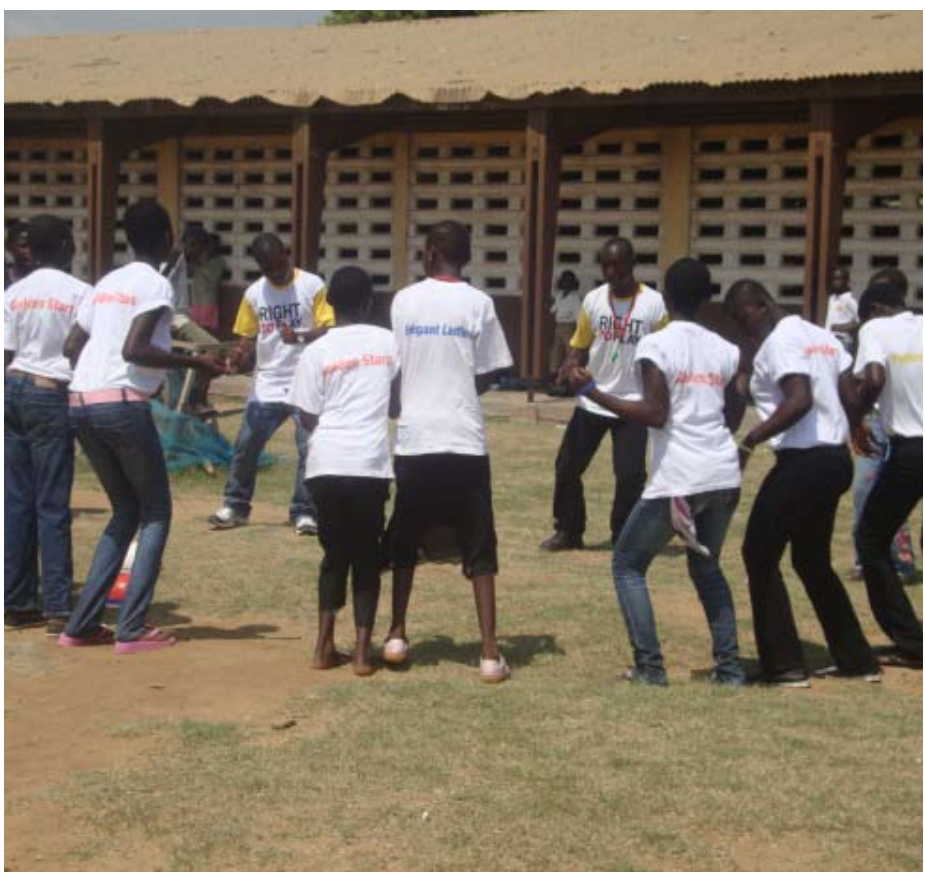

Right to Play ${ }^{\odot}$ encourage les filles à participer à des activités et à des jeux qui développent les compétences de leadership et de coopération en liaison avec l'éducation à la santé. 
Les 90 adolescentes se réunissent deux fois par semaine en groupes de 20 à 25 filles. Les séances sont dirigées par des tutrices et se concentrent sur le développement des compétences de base dans les domaines suivants : compétences de la vie courante, santé générale, santé sexuelle et reproductive et capacités financières. Parallèlement à ce programme, les filles sont présentées à des ressources et à des services pertinents au sein de leur communauté. Les filles apprennent l'estime de soi et l'auto-identité au cours de la séance sur la vie courante et reçoivent des cartes d'identification au programme. Ces cartes aident les filles à se reconnaître en tant que citoyennes et les encouragent à demander des documents supplémentaires qui pourraient leur permettre d'accéder à des droits sociaux. Pendant les séances consacrées à la santé, les participantes au programme visitent le centre de santé communautaire pour acquérir des informations importantes pour la santé et connaître les services à leur disposition. En outre, les filles visitent une entreprise locale au cours de la séance sur les capacités financières pour connaître les possibilités génératrices de revenus disponibles dans leur communauté.

Ce programme pilote est conclu en partenariat avec Right to Play ${ }^{\odot}$, qui utilise l'activité physique pour fournir des informations clés aux jeunes gens. Les tutrices du programme sont formées à des activités et à des jeux qui développent les compétences en leadership et en coopération en liaison avec l'éducation à la santé. Les tutrices intègrent plusieurs de ces activités dans leurs séances avec les groupes de filles et les premières réactions sont très encourageantes, notamment parce que les adolescentes du quartier U-Compound ont des possibilités limitées de pratiquer des activités récréatives.

Pendant la durée de la phase pilote, l'équipe du projet évaluera les aspects du programme qui fonctionnent bien et ceux nécessitant des améliorations avant d'élargir le programme à un plus grand groupe de filles. Les résultats de cette évaluation seront partagés avec la communauté et contribueront à l'élaboration de la prochaine phase du programme.
Les premières observations du projet pilote révèlent que les parents accordent souvent la priorité aux garçons en matière de scolarisation et que les adolescentes sont souvent déscolarisées pour gagner de l'argent dans le secteur informel. Cette déscolarisation se produit à l'âge de la puberté pour les filles. C'est pourquoi l'équipe du projet travaillera avec les filles âgées de 10 à 13 ans au cours de la prochaine phase du projet. Ce changement permettra de renforcer les ressources des filles avant qu'elles n'entrent dans une période critique d'évolution de leur développement.

\section{Bibliographie}

Austrian, Karen et Dennitah Ghati. 2010. Girl Centered Program Design: A Toolkit to Develop, Strengthen and Expand Adolescent Girls Programs. New York: Population Council.

Brady, Martha. 2003. "Chapter 7-Safe spaces for adolescent girls," in Adolescent and Youth Sexual and Reproductive Health: Charting Directions for a Second Generation of Programming - Background Document for the Meeting. New York: UNFPA, pp.155-176.

Population Council. 2010. "The Adolescent Experience In-Depth-Using Data to Identify and Reach the Most Vulnerable Young People: Ghana 2008." New York.

Population Reference Bureau (PRB). 2010. "Fiche de données sur la population mondiale 2010." Washington, DC. http://www.prb.org/FrenchContent/ Articles/2010/2010wpds_fr.aspx Consulté le 6 mai 2011.

\section{Donateurs}

Ministère britannique du développement international (DFID)

Institutions partenaires:

Right to Play ${ }^{\odot}$

Service de santé du Ghana

\section{Population Council}

Le Population Council fait évoluer les perspectives sur les questions essentielles de santé et de développement. Nous cherchons à comprendre les causes et les conséquences de l'inégalité entre les sexes et des disparités sociales qui s'installent au cours de l'adolescence. Nous fournissons des données en vue d'améliorer les programmes et les politiques mis en place sur le terrain qui permettent d'assurer des transitions efficaces et productives vers la vie adulte dans les pays en développement. www.popcouncil.org

(c) 2011 The Population Council, Inc. 